\title{
Research of Sensor Fault-Tolerant Control Based on Analytical Reconstruction Model for Electronic Brake Pedal Simulator
}

\author{
Ying-Lan Yu, Yi-Hu Xu, and Yi-Nan Xu
}

\begin{abstract}
Electronic brake pedal simulator is the core component of pure electric vehicle braking system, affecting braking and safety performances directly. In this paper, a mathematical model of the electronic brake pedal simulator is established according to the physical structure and motion characteristics of that. A sensor fault diagnosis method based on particle filter algorithm is proposed for the angular displacement sensor and pedal force sensor in electronic brake pedal simulator. And an adaptive fault-tolerant control architecture model is established by applying the mathematical model of the electronic brake pedal simulator and the sensor fault diagnosis model. The SW/HW simulation results show that the fault diagnosis and adaptive fault-tolerant control method can accurately detect sensor faults. In a sensor failure, the adaptive fault-tolerant control architecture model estimates the sensor output value in the normal state, which makes electronic brake pedal emulator can still operate normally, so as to further improve the reliability of electronic brake pedal simulator.
\end{abstract}

Index Terms - Electronic brake pedal simulator, sensor fault, particle filter, adaptive fault-tolerant control.

\section{INTRODUCTION}

Electro-mechanical brake (EMB) system based on drive-by-wire technology not only has the feasibility but also become the development direction of the next generation of automobile brake system with the accelerating development of automobile electronic technology [1], [2]. Contrasting with the conventional Hydraulic braking (HB) system, the EMB system is based on electronic components instead of the hydraulic components and pipes. EMB system is an electro-mechanical integration system, which is mainly composed of electronic brake pedal simulator, electronic control unit (ECU), vehicular communication networks, EMB actuators, EMB controller and various sensors [3]. The EMB system has advantages such as small volume, high efficiency, simple structure, convenient installation \& maintenance, flexible arrangement and the active adaptability for different drivers in different running conditions.

Electronic brake pedal simulator is the bridge between the driver and EMB actuator, which can provide the driver brake pedal feel similar to that of the traditional brake system. A good brake pedal feeling not only can enhance driving

Manuscript received October 20, 2015; revised December 18, 2015. This work was supported by the National Natural Science Foundation of China (61361003).

The authors are with the College of Engineering of Yanbian University, Yanji, 133002, China (e-mail: lgjw@ybu.edu.cn, xuyh@ybu.edu.cn, ynxu*@ybu.edu.cn). comfort and safety, but also is the main subjective evaluation factor for the driver for pure electric vehicle braking system performance, so the performance of electronic brake pedal simulator has important significance. Electronic brake pedal simulator consists of pedal feel simulator, pedal sensors, pedal and the support frame. Once the sensor failure, the driver's braking intention can't be described correctly, it will cause the failure of the whole EMB system, and even will lead to traffic. So the electronic brake pedal simulator should have the ability of fault diagnosis and fault tolerance.

In the conventional research of fault detection and diagnosis technology, one kind of fault diagnosis method based on Kalman filter was proposed against sensor failures [4]. This method can effectively detect the sensor fault. However, the controlled system is linear system, and there is a big difference between the actual nonlinear systems. For nonlinear systems, reference [5], establishes a fault detection and fault-tolerant control model based on neural network algorithm, which could detect and isolate faults in time. However, the neural network algorithm needs a large number of training samples, has a complex structure, and shows poor real-time performance, so it is difficult to meet actual goals of electric control system. A conventional research for the electronic brake pedal simulator, reference [6], proposed a fault diagnosis method based on width detection and frequency detection according to the electrical characteristics of electronic pedal. This method can detect sensor failure that does not satisfies electrical properties of electronic pedal, but cannot detect gradual fault. In reference [7], a fault diagnosis method adopting threshold and correlation alliance was proposed aiming at detecting electronic pedal sensor fault. However, such a method is suitable for abrupt faults only, and unable to detect slow variation faults. According to above analysis results, current researches about sensor fault diagnosis and fault-tolerant control mainly focus on single type of fault, and lack multiple type of faults diagnosis in nonlinear generalized systems.

In order to accurately describe the driver's brake intention, we proposed an adaptive fault-tolerant control method based on analytic reconstruction model. And this method helps electronic brake pedal simulator still run normally under the condition of sensor malfunction. In Section II, a mathematical model is established based on the physical structure and motion characteristics of the electronic brake pedal simulator. And a kind of fault detector for angular displacement and force sensor is established by adopting particle filter algorithm in Section III. In Section IV, the adaptive fault-tolerant control architecture model of electronic brake pedal simulator is proposed based on the 
mathematics model and sensor fault diagnosis model of the electronic brake pedal simulator. In Section V, the feasibility of fault detection and fault tolerant-control is verified by SW/HW simulations. Finally, brief conclusions are given in Section VI.

\section{The Mathematical Model of EleCtronic BRAKE PEDAL SiMULATOR}

The structure diagram of electronic brake pedal simulator is shown in Fig. 1. Electronic brake pedal simulator mainly consists of pedal feel simulator, pedal sensors, pedal and the support frame.

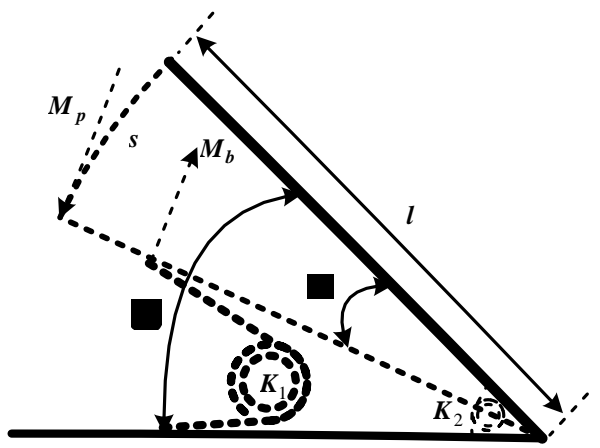

Fig. 1. Electronic brake pedal simulator structure diagram.

In Fig. 1, $\alpha$ measured by the angular displacement sensor represents the angular displacement of the pedal when electronic brake pedal simulator is at work. $F_{p}$ measured by pressure sensor represents pedal force. And torsional spring $K_{1}$ and $K_{2}$ are used to simulate the pedal feel and make the pedal back in situ when the driver loosen the pedal.

The motion equation of the pedal is given by equation (1) based on motion characteristics of electronic brake pedal under the condition of inertia.

$$
J \ddot{\alpha}=M_{p}-\left[M_{b}-\left(k_{2} \alpha+d \dot{\alpha}\right)\right]
$$

$\alpha, M_{p}, M_{b}, K_{2}, d, j$ represent angular displacement, human input torque added to the pedal, reaction torque from the pedal, spring constant, attenuation constant and inertia, respectively.

According to the equation (1), the state equation of electronic brake pedal simulator is derived as:

$$
\begin{gathered}
\left\{\begin{array}{c}
\dot{x}=A x+B u \\
y=C x
\end{array}\right. \\
\text { where, } x=\left[\begin{array}{c}
\dot{\alpha} \\
\ddot{\alpha}
\end{array}\right], A=\left\lfloor\begin{array}{cc}
0 & 1 \\
-\frac{k}{J} & -\frac{d}{J}
\end{array}\right\rfloor, B=\left[\begin{array}{l}
0 \\
1
\end{array}\right], C=\left[\begin{array}{ll}
1 & 0 \\
0 & 1
\end{array}\right]
\end{gathered}
$$

According to the physical structure of the electronic brake pedal simulator, the relationship between the pedal force and torque is given as equation (3). $F_{p}$ and $l$ represent human input force added to the pedal and the pedal length, respectively.

$$
M_{P}=F_{P} l
$$

\section{Sensor Fault Detection Based on Particle Filter ALGORITHM}

EMB controller determines the driver's brake intention by receiving pedal angular displacement signal and pedal force signal. Obviously, once the sensor failure, there will be no detection of driver's brake intention and no reliable breaking effect, and even cause the failure of brake system. Therefore it is necessary to detect faults for sensor and estimate normal output signal of faulty sensor through the fault-tolerant control architecture model when the sensor fails. Hardware redundancy and analytical redundancy are usually used in the research of sensor fault diagnosis. Hardware, redundancy method can monitor and detect fault in real time, and effectively isolate faulty sensors. However it needs more components and high cost. Analytical redundancy technology makes up the shortcoming of the hardware redundancy. Therefore, it becomes the development direction of sensor fault diagnosis and fault tolerance [8].

Particle filter (PF) is a nonlinear estimation method based on analytic signal processing. It abandons the restriction condition that random quantity must satisfy Gaussian distribution when solving the nonlinear filtering problem, and can deal with strongly nonlinear, non-Gaussian random dynamic systems [9], [10]. In this paper, particle filter was adopted to construct a fault detection model for malfunction of two kinds of sensors carried in electronic brake pedal simulator on the basis of the proposed mathematical model of electronic brake pedal simulator. Its principle is shown in Fig. 2.

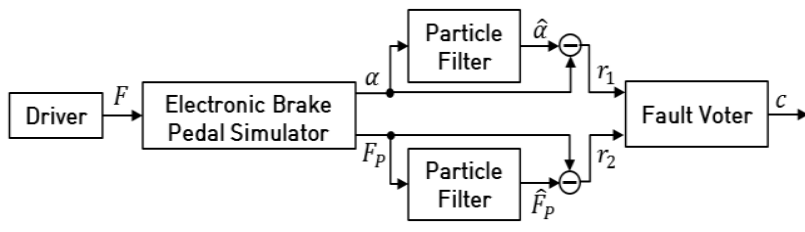

Fig. 2. Sensor fault diagnosis principle of electronic brake pedal.

When the driver presses the pedal, angular displacement $\alpha$ and pedal force $F_{p}$ will be output to particle filters by electronic brake pedal simulator. Then the angular displacement estimation $\hat{\alpha}$ and pedal force $\hat{F}_{p}$ estimation will be produced, respectively. Fault detection of sensors will be realized by residual values of estimation output and real output of sensors. The expressions of residual value are as follows:

$$
\begin{aligned}
& r_{1}=a-\hat{a} \\
& r_{2}=F_{P}-\hat{F}_{P}
\end{aligned}
$$

Because of its poor work environment, electronic automobile sensor system will be affected by outside factors such as humidity, vibration, electromagnetic fields and may appear all kinds of faults. In order to improve the reliability of electronic brake pedal simulator model, considering the 
influence of outside noise, the electronic brake pedal simulator model proposed above can be expressed as follows:

$$
\begin{aligned}
& x_{k+1}=f\left(k, x_{k}, u_{k}\right)+w_{k} \\
& y_{k}=h\left(k, x_{k}\right)+v_{k}
\end{aligned}
$$

where, $x_{k}$ is a discrete system state variable, $u_{k}$ is the input control variable, $y_{k}$ is the system output observation, $w_{k}$ and $v_{k}$ are process noise and observation noise, respectively. $f(\cdot)$ and $h(\cdot)$ are nonlinear functions.

When the electronic brake pedal simulator is working normally, the possible fault characteristics can be described as follows:

$$
\begin{aligned}
& x_{k+1}=f\left(k, x_{k}, u_{k}\right)+w_{k} \\
& y_{k}=h\left(k, x_{k}\right)+g\left(s_{k}\right)+v_{k}
\end{aligned}
$$

where, $g\left(s_{k}\right)$ is a sensor fault function.

Simulated electronic brake pedal simulator by particle filters can be expressed as:

$$
\begin{aligned}
& \hat{x}_{k+1 \mid k}=f\left(k, \hat{x}_{k \mid k}, u_{k}\right) \\
& \hat{y}_{k \mid k}=h\left(k, \hat{x}_{k \mid k}\right)
\end{aligned}
$$

where $\hat{x}_{k / k}$ is the optimal estimate of $x_{k}$.

Compare equation (6) with (7), assuming that filter has stabilized at time $K$, the state estimation is equal to the real state, state estimation error tends to be zero, then the output residuals are:

$$
r_{k}=y_{k}-\hat{y}_{k \mid k}=g\left(s_{k}\right)+v_{k}
$$

When the sensor is working normally, the mean value of the residual tends to zero, otherwise it is not equal to zero. Considering the uncertainty of model error, noise interference and so on, an appropriate threshold needs to be set. When the absolute value of the residual value is greater than the threshold, the sensor fault can be determined.

\section{An AdAPTIVE FAUlT-TOLERANT CONTROL ARCHITECTURE MODEL BASED ON ANALYTIC RECONSTRUCTION MODEL}

When fault detector detect the faults of sensor, it is necessary to isolate the fault sensors and establish the fault reconstruction model for faulty sensors. The output signals of faulty sensor can make use of the mathematical model of electronic brake pedal simulator and signals of normal sensor to estimate. Due to the short displacement process of electric brake pedal simulator, the impact of the angular velocity and Angle acceleration pedal is small, so the angular velocity and Angle acceleration pedal can be ignored when describing the relationship between the pedal force and angular displacement. According to equation (1), the reconstruction models of the angular displacement sensor and pedal force sensor can be built.

$$
\begin{aligned}
& \hat{\alpha}_{1}=\frac{-M_{p}+M_{b}}{k_{2}} \\
& \hat{M}_{P}=M_{b}-k_{2} \alpha
\end{aligned}
$$

Fig. 3 shows the adaptive fault-tolerant control architecture, which is set up through the fault detection model of electronic brake pedal simulator and reconstruction models of two sensors.

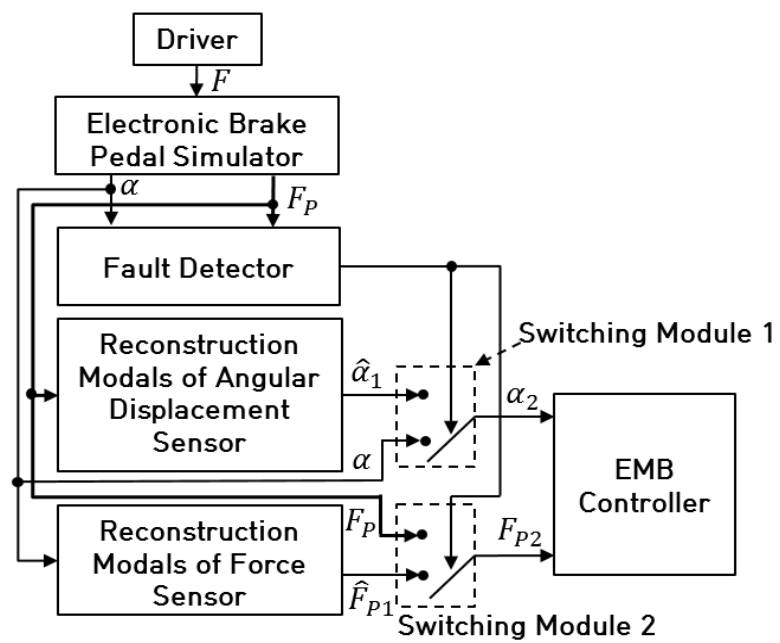

Fig. 3. Adaptive fault-tolerant control architecture model for electronic brake pedal simulator.

In Fig. 3, the electronic brake pedal simulator outputs pedal force $F_{p}$ and angular displacement $\alpha$ after receiving the driver's input force $F . F_{p}$ is input to the fault detector and the angular displacement reconstruction modal, and $\alpha$ is input to the fault detector and the pedal force reconstruction model. The fault detector is used to detect two kinds of sensor failures, and the reconstruction modals of angular displacement sensor and pedal force sensor are used to estimate the angular displacement sensor output values $\hat{\alpha}_{1}$, and pedal force sensor output values $\hat{F}_{p 1}$, respectively. The inputs of signal switching module are the actual value of sensors and the estimated values of reconstruction model. And the outputs of signal switching module are controlled by the control signal $c$. When angular displacement sensor is trouble-free, the outputs of the signal switching module 1 are actual sensor signal, that is $a_{2}=a$; when the angular displacement sensor fails, $a_{2}=\hat{a}_{1}$. When pedal force sensor is trouble-free, the outputs of the signal switching module 2 is the actual sensor signal, that is $F_{P 2}=F_{P}$, when the pedal force sensor fails, $F_{P 2}=\hat{F}_{P 1}$. Eventually the output value of the corresponding sensor is sent to the EMB controller.

\section{Simulation VerificAtion}

\section{A. Matlab Simulations}

In order to validate reliability of the proposed adaptive fault-tolerant control method, the mathematical model of the electronic brake pedal simulator has been set up in the 
Matlab/Simulink environment. The simulation parameters are shown in Table I.

TABLE I: SIMULATION PARAMETERS FOR ELECTRONIC BRAKE PEDAL

\begin{tabular}{ccc}
\hline symbol & \multicolumn{1}{c}{ parameter } & value \\
\hline$k 1$ & Torsion spring coefficient & 79.36 \\
$k 2$ & Reset spring coefficient & 78.03 \\
$d$ & Attenuation coefficient & 0.8701 \\
$J$ & Inertia coefficient & 0.14231 \\
\hline
\end{tabular}

A braking cycle $(30 \mathrm{~ms})$ of sensor signal is chosen as a sampling example, and the rectangular wave is adopted to simulate fault occurred in a sensor at 10 to $20 \mathrm{~ms}$. The simulation results are shown in Fig. 4 and Fig. 5.

Fig. 4 and Fig. 5 show fault detection and fault-tolerant control simulation results of angular displacement sensor and that of pedal force sensor, respectively. In Fig. 4 and Fig. 5, (a) is the comparison result of measured and estimated values; (b) is the comparison result of measured and reconstructed values.
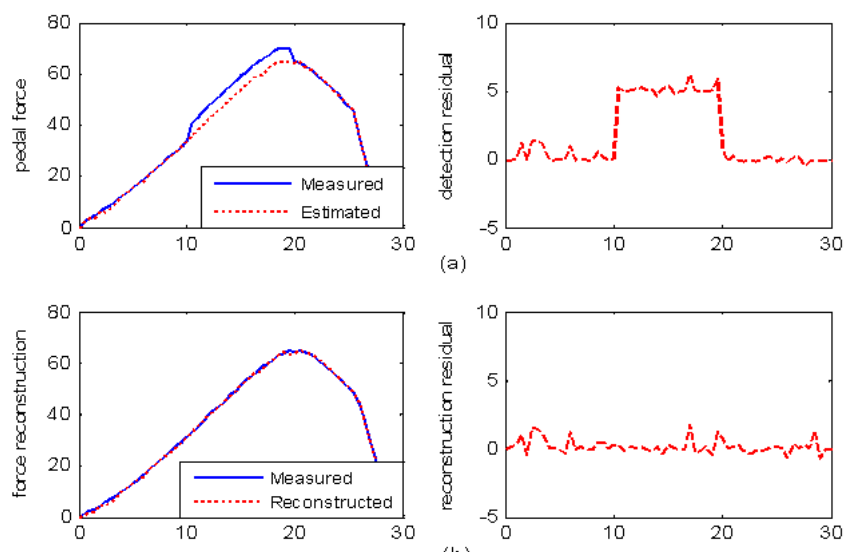

(b)

Fig. 4. Angle detection and tolerance for force sensor: (a) angle sensor fault diagnosis; (b) angle sensor signal reconstruction.
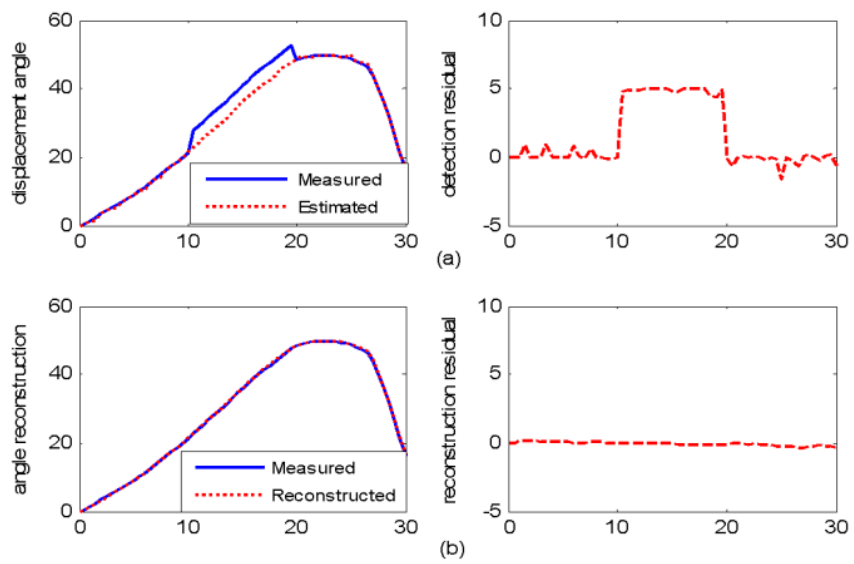

Fig. 5. Fault detection and tolerance for force sensor: (a) force sensor fault diagnosis; (b) force sensor signal reconstruction.

From simulation results, when a sensor is trouble-free, there is no difference between the estimated signals from the particle filter and real signals from the sensor. When a sensor fault occurs at $10 \mathrm{~ms}$, it is observed that there is an obvious jump between actual measured values and estimated values. The residual wave shows that the change rule of residuals is corresponding to the characteristics of sensors faults mostly. It shows that fault detector based on particle filter algorithm can quickly detect sensor fault and accurately locate faulty sensor. At the same time, the reconstruction model of a sensor will not be affected by faulty sensors and accurately estimates output signals of the sensor in normal working state.

\section{B. Hardware in the Loop Simulation (HILS)}

In order to further verify the reliability and practicability of the proposed adaptive fault-tolerant control method, the hardware in the loop experiment is realized by using commercial electronic brake pedal simulator. The block diagram of hardware test environment is shown in Fig. 6. The pedal angle displacement signal and the pedal force sensor signal pass through the amplifier and A/D converter to obtain digital signals of two kinds of sensors, which will be inputs to the commercial electronic automatic pedal simulator. In order to achieve the goal of integrated control based on the hardware system, this paper realizes the fault diagnosis module and fault-tolerant control module by using ARM processor and FPGA. So then, the digital signals of the sensors are inputs to the fault diagnosis unit and the fault tolerant-control unit. The final outputs of the pedal angular displacement and pedal force sensor signal are converted to an oscilloscope via D/A converter. The actual realization of test environments is shown in Fig. 7.

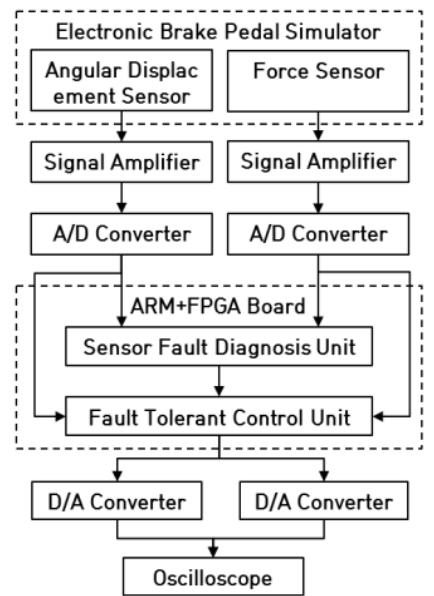

Fig. 6. Block diagram of hardware test environments.

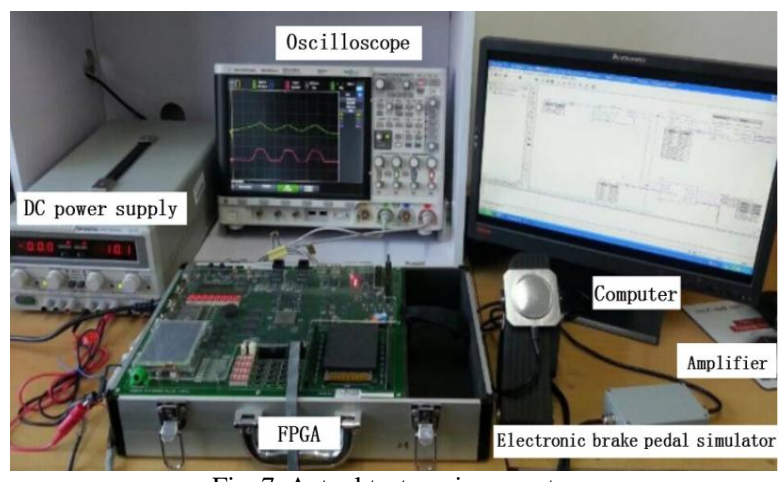

Fig. 7. Actual test environment.

Fig. 8 and Fig. 9 show the hardware simulation results of the pedal angle displacement sensor and that of pedal force sensor, respectively. In Fig. 8 and Fig. 9, (a) is the actual output value when the sensor is trouble-free; (b) is the actual output value when the sensor is on normal operation at 0 to $3.1 \mathrm{~s}$, but the sensor fault occurs at $3.1 \mathrm{~s}$, so the output signal is zero; (c) is the fault tolerant-control output value. It shows that the system accurately estimates the output value after the 
fault was detected at $3.1 \mathrm{~s}$.

The above experimental results show that the proposed fault detection and fault tolerant-control methods are still valid.

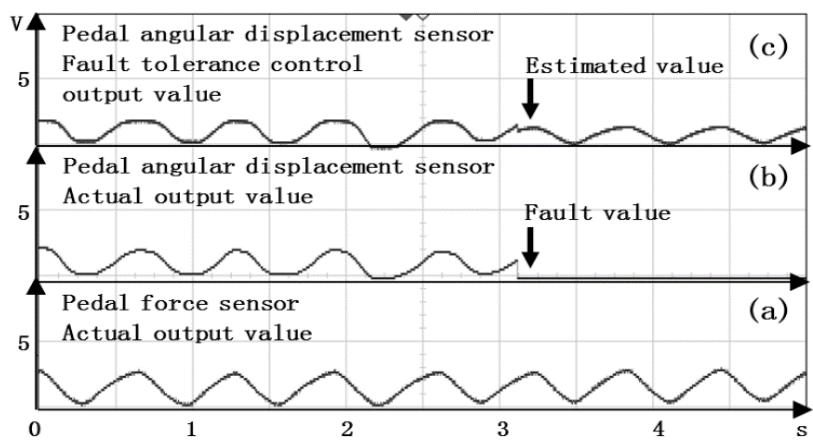

Fig. 8. Hardware simulation results of pedal angle displacement sensor.

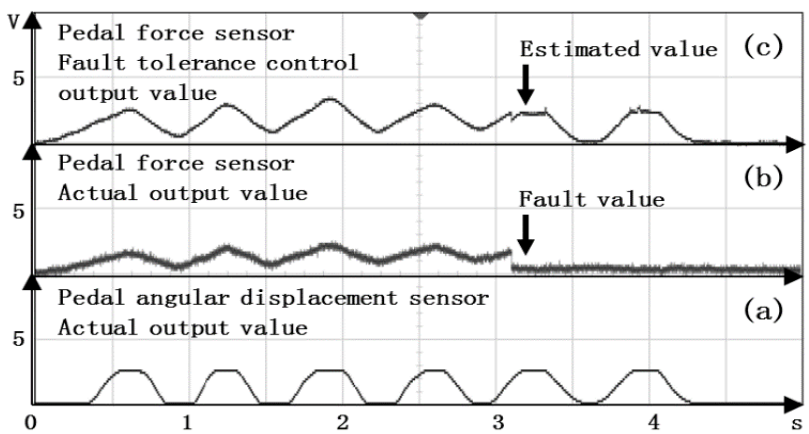

Fig. 9. Hardware simulation results of pedal force sensor.

\section{CONCLUSION}

In EMB system, the electronic brake pedal simulator is used to transmit the driver's braking intention so its performance directly affects safety and reliability of the automobile braking system. In this paper, a mathematical model of electronic brake pedal simulator is established. Considering nonlinear effects on the system, the fault detectors based on particle filter algorithm are established for the pedal angular displacement sensor and force sensor, respectively, which can effectively detect sensor faults. At the same time, an adaptive fault-tolerant architecture model based on analytic reconstruction of sensors is established. When the fault of a sensor is detected, the reconstruction model of fault sensor will accurately estimate normal values of sensor. Consequently, the reliability of the electronic brake pedal simulator is improved.

\section{REFERENCES}

[1] J. K. Ahn, K. H. Jung, D. H. Kim et al., "Analysis of a regenerative braking system for hybrid electric vehicles using an electro-mechanical brake," International Journal of Automotive Technology, pp. 229-234, 2009.
[2] A. Dardanelli, G. Alli, and S. Savaresi, "Modeling and control of an electro-mechanical brake-by-wire actuator for a sport motorbike," Mechatronic Systems, pp. 524-531, 2010.

[3] B. D. Choe, W. H. Hwang, and K. S. Huh, "Modeling of EMB (electro mechanical brake) to emulate gearbox fault and control," Transactions of the Korean Society of Automotive Engineers, pp. 33-38, 2012.

[4] G. H. B. Foo, X. Zhang, and D. M. Vilathgamuwa, "A sensor fault detection and isolation method in interior permanent-magnet synchronous motor drives based on an extended Kalman filter," IEEE Transactions on Industrial Electronics, pp. 3485-3495, 2013.

[5] C. W. Chen, P. C. Chen, and W. L. Chiang, "Modified intelligent genetic algorithm-based adaptive neural network control for uncertain structural systems," Journal of Vibration and Control, pp. 1333-1347, 2013.

[6] C. Wang, T. Zhang, H. S. Yuet et al., "Research on electronic pedal signal collecting and fault diagnosis strategy," Vehicle and Power Technology, pp. 49-52, 2010.

[7] C. Liu, C. F. Zong, J. Liu, and F. Sha, "An experimental study on fault diagnosis algorithm of electronic pedal modules in in-wheel motor driven electric vehicles," Computer Measurement and Control, pp. 2913-2915, 2012.

[8] S. Nandi, H. A. Toliyat, and X. D. Li, "Condition monitoring and fault diagnosis of electrical motors-a review," IEEE Transactions on Energy Conversio, pp. 719-729, 2005.

[9] J. Yu, "A particle filter driven dynamic gaussian mixture model approach for complex process monitoring and fault diagnosis," Journal of Process Control, pp. 778-788, 2012.

[10] J. F. Daum, "Nonlinear filters: Beyond the Kalman filter," Aerospace and Electronic Systems Magazine, pp. 57-69, 2005.

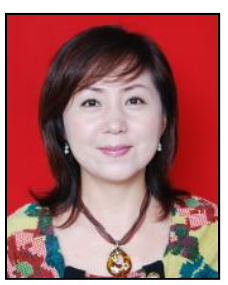

Ying-Lan Yu was born in Jilin province of China. She received the master course in western philosophy from the Yanbian University, China, in 2009.

She is a lecturer of College of Engineering of Yanbian University, Yanji, China.

Her research interests include the fault diagnosis and fault-tolerant control.

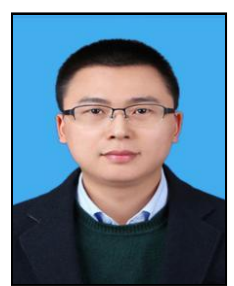

Yi-Hu Xu was born in Jilin province of China. He received the Ph.D. degree in electronics engineering from the Chonbuk National University, Korea, in 2014.

$\mathrm{He}$ is a lecturer of the division of electronic and communication engineering of Yanbian University, Yanji, China.

His research interests include the automobile electronic control and network.

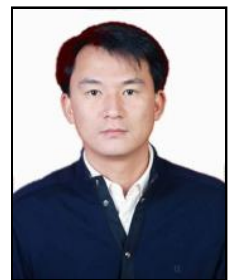

Yi-Nan Xu was born in Jilin province of China. Currently, he is an associate professor of the division of electronic and communication engineering of Yanbian University, Yanji, China.

$\mathrm{He}$ received the Ph.D. degree in electrical engineering from the Chonbuk National University, Korea, in 2009.

His research interests include the in-vehicle networks and automobile electronic control. 